\title{
Analytical evaluation of fats and oils by size-exclusion chromatography
}

\author{
M.C. Dobarganes and G. Márquez-Ruiz \\ Instituto de la Grasa, Avda. Padre García Tejero 4, 41010 Sevilla, Spain
}

In this paper, the most recent applications of high-performance size-exclusion chromatography (HPSEC) in analyses of fats and oils are presented. Directly used in oils, the technique shows utility for evaluation of polymers in used frying fats and oxidized samples, as well as for analysis of low-caloric fats in blends. In concentrated fractions of polar compounds, characterization of virgin and refined oils and follow-up of early oxidation stages are also attainable. An extended application to fatty acid derivatives allows complementary analyses of specifically oxidized and polymeric fatty acyl groups.

\section{Characteristics of HPSEC in oil analysis}

Separation by exclusion chromatography is based on the relative molecular size of compounds, normally related to their molecular weight (MW), provided that compounds have similar shape. Among its advantages, simplicity, rapidity and high repeatability are worthy to stand out. Typically, stationary phases consist in macromolecules cross-linked to form a three-dimensional network characterized by a specific pore size. The most important parameters influencing resolution are the pore volume, pore-size distribution and particle size. For applications of HPSEC in organic solvents directed to analyses of fats and oils, copolymers of styrene divinyl benzene are generally used, being 50, 100 and $500 \AA$ essential porosities for separations in the range $100-20000 \mathrm{MW}$, and particle sizes of 5 and $10 \mu \mathrm{m}$ are the most common. As to the mobile phase, a single solvent is used and its selection is limited by the solubility of the sample. Although toluene and dichloromethane are selected for certain applications, tetrahydrofuran is the most popular solvent and flow rates between 0.5 and $1.5 \mathrm{~mL} / \mathrm{min}$ permit analyses to be completed in less than $30 \mathrm{~min}$. For quantitative purposes, the refractive index and evaporative light-scattering detectors are generally used, especially the former because of its simplicity, i.e., linear responses in the ranges of interest. General characteristics of HPSEC have been described in detail in recent reviews [1-3].

\section{Applications}

\section{Total oil samples}

Evaluation of high MW compounds by HPSEC directly in entire oil samples constitutes an excellent application of the technique for its simplicity and rapidity, and it is of great utility for certain fields of lipid analysis. It is only necessary to dilute the fat or oil in the appropriate solvent and chromatographic analysis is performed in just 10-30 min, depending on the flow rate and number of columns. In this context, HPSEC has been satisfactorily used for analyses of polymers in used frying fats [4] and in the area of fish oils for evaluation of oxidative polymerization products [5]. Additionally, applications can be extended to analyses of fat substitutes in blends provided that MWs of the compounds involved differ significantly.

\section{a) Analysis of polymers in used frying oils}

Direct application of HPSEC is of special relevance for quality evaluation of used frying fats, where polymers are the most representative group of compounds due to thermoxidative alteration. Hence the IUPAC Commission on Oils, Fats and Derivatives has recently published results of two interlaboratory tests and decided to adopt the method although limiting applications to samples containing $3 \%$ or more of polymerized triglycerides [6].

The method proposes a single column of $30 \mathrm{~cm} \times$ $0.77 \mathrm{~cm}$ i.d. packed with copolysterene divinyl benzene (5 $\mu \mathrm{m}$ particle size), tetrahydrofuran as the mobile phase, a refractive index detector, sample concentration of $50 \mathrm{mg} / \mathrm{mL}$ for an injection valve with a $10 \mu \mathrm{L}$ loop and a flow rate of $1 \mathrm{~mL} / \mathrm{min}$. Under such conditions, the analysis time is about $10 \mathrm{~min}$.

\section{b) Detection of the onset of advanced oxidation stages}

Lately, we have found an interesting application of direct analysis of oil samples by HPSEC, other than that related with heated fats. It is well-known the great demand for analytical methods to monitor oxidation during storage that help determine the shelf-life of oils and fatty foods. In this context, a number of experiments have been undergone in our laboratory using model systems such as trilinolein and triolein, at low and moderate temperature. From the results obtained, it was generally observed that at a certain point in the course of oxidation samples enter an accelerated phase, characterized by a sharp increase of primary oxidation products and inititation of polymerization. Defining the induction period as the time interval before oxidation proceeds rapidly, a significant rise in polymers hence denotes the end of the induction period and the onset of the advanced oxidation stage [7].

This approach is currently been used to determine the shelf-life of microencapsulated fish oils as dried powders, a potential food ingredient directed to diet supplementation with polyunsaturated fatty acids, wherein the oil is surrounded by a matrix composed of carbohydrates and proteins so as to gain stability. As an illustrative example, 
figure 1 shows evolution of oxidation of microencapsulated oils prepared by freeze-drying during storage at ambient temperature in light and in the dark, wherein changes in triglyceride polymers are represented. At a certain point, a significant rise in polymers is observed, around 10 and 37 days for samples in light and in the dark, respectively, indicating the end of the induction period and, interestingly, these results were in excellent agreement with parallel sensory assessment showing that samples were distinctly rancid from 12 days in light and 37 days in the dark.

\section{c) Evaluation of fat substitutes in blends with natural} fats or oils

HPSEC use has been extended to lipid samples other than classical fats and oils. Development of low-caloric fats to be used in baked and fried fatty foods has received much attention in the last years and, among the fat substitutes proposed, sucrose polyesters (SPE), i.e., sucrose molecules esterified by $5-8$ fatty acids, probably stand out and have been recently approved for use in foods by the U.S. Food and Drug Administration. Hence the need for valid analytical methods applicable to the evaluation of mixtures of SPE and natural oils in commercialized products, of enormous interest for production and quality control, and nutritional labeling [8].

Recently, we suggested the utilization of HPSEC to characterize SPE/triglyceride mixtures [9] and later obtained satisfactory quantitative results for SPE/natural oil blends [10]. Figure 2 illustrates the efficacy of the HPSEC separation for olive oil SPE/sunflower oil mixtures containing 17.1 and $26.7 \%$ SPE. HPSEC served not only to quantitate the relative proportions of both parts in the mixture, which is essential to define the caloric value of the product, but also to know the fatty acid composition of each component following recovery of the separately eluted fractions, of additional nutritional interest given that exclusively the fraction of natural fat or oil can be digested and absorbed.

Similarly, the utility of HPSEC can be extended to the analysis of mixtures of natural oils with other fat substitutes differing significantly in MW, such as sorbitol polyesters, trehalose polyesters or raffinose polyesters.

\section{Fractions of polar compounds}

Polar compound determination based on adsorption chromatography was proposed for quality evaluation of used frying fats given that during frying a complex mixture of degradation products of higher polarity than that of the original triglycerides are formed. Thus, quantitation of polar compounds content by silica columns [11] was standardized by the IUPAC and nowadays constitutes the basis of some European regulations to discard used frying fats for human consumption [12]. Apart from this application, separation of polar fractions results in substantial advantages for further quantitation by HPSEC of all groups of minor compounds because of the concentration effects. In this context, we have developed methodologies based on a combination of adsorption and size exclusion chromatographies and found useful applications not only in heated fats but also for quality evaluation of refined oils, characterization of virgin oils and follow-up of early oxidation stages.

\section{i) Combination silica columns-HPSEC}

This analytical procedure was developed with the aim of obtaining fairly complete information on the types of degra-

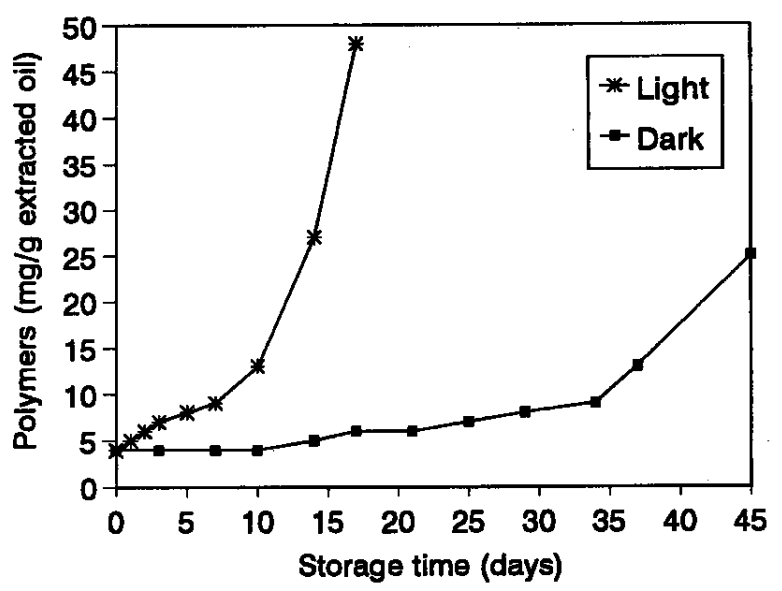

Figure 1. Evolution of triglyceride polymers in microencapsulated fish oils stored at room temperature.

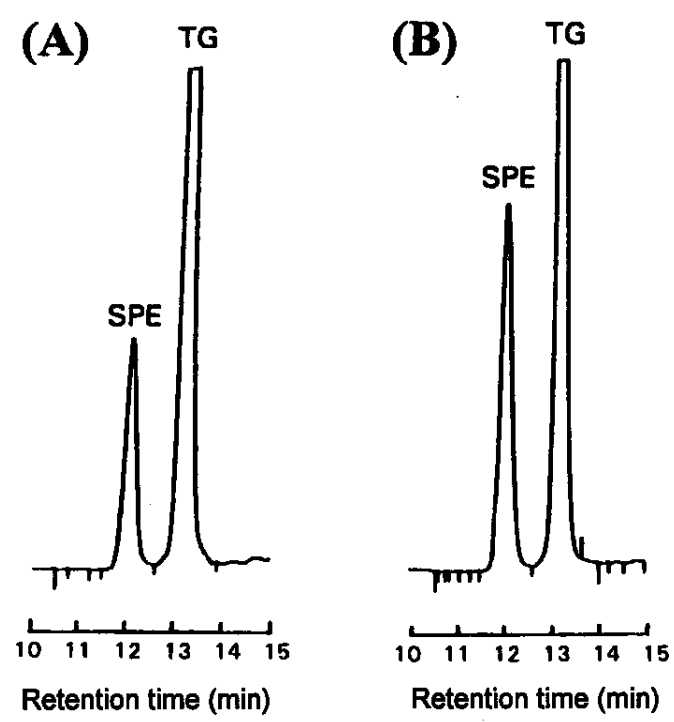

Figure 2. HPSEC chromatograms of olive oil sucrose polyesters/sunflower oils mixtures. (A) $17.1 \%$ SPE and (B) $26.7 \%$ SPE. Abbreviations: SPE, sucrose polyesters; TG, triglycerides.

dation in used frying oils [13]. It consists on conveniently combining the two methods proposed by IUPAC $[6,11]$, namely gravimetric determination of polar compounds by silica columns starting from $1 \mathrm{~g}$ samples, eluting nonpolar and polar fractions with $150 \mathrm{~mL}$ of a mixture hexane:diethyl ether 90:10 and $150 \mathrm{~mL}$ of diethyl ether, respectively, plus further analysis of polar fractions by HPSEC. 100 and $500 \AA$ columns with polysterene divinylbenzene highly cross-linked macroporous packing (particle size: $5 \mu \mathrm{m}$ ) connected in series, tetrahydrofuran as mobile phase (flow rate: $1 \mathrm{~mL} / \mathrm{min}$ ) and a refractive index detector were used.

\section{ii) Combination solid phase extraction-HPSEC}

A modification of the former methodology has been introduced in order to reduce quantity of sample and solvents and shorten analysis time. Briefly, starting from just $50 \mathrm{mg}$ of sample and using alternatively silica cartridges $(1 \mathrm{~g})$, $15 \mathrm{~mL}$ of elution solvents are used for each fraction. For 
quantitative purposes, monostearin (a solution aliquot containing $1 \mathrm{mg}$ ) is added as internal standard given that gravimetric determination would provide poor reproducibility. The procedure is useful for samples within a wide range of alteration and especially adequate for those of low oxidation level, showing significantly lower relative standard deviations than gravimetric determination [14].

Figure 3 shows HPSEC chromatograms of an used frying oil and its polar fraction, this latter resulting in enhanced sensitivity for quantitation of all groups of alteration compounds.

\section{a) Analysis of used frying fats and oils}

The methodology based on a combination of silica columnsHPSEC has been revealed as an excellent alternative for used frying oil evaluation [13,15]. First, a substantial increase in quantitation possibilities of polymers is achieved, overcoming the limitation of $3 \%$ content established for analyses in total samples. Also, the independent determination of oxidized triglyceride monomers from unoxidized triglycerides eluting in the non-polar fraction offers a measurement of oxidative deterioration and finally, simultaneous evaluation of diglycerides constitutes an indication of hydrolytic alteration, avoiding any overlap with the most abundant triglyceride peak in the whole sample.

\section{b) Quality evaluation of refined oils}

Possibilities of HPSEC for quality assessment of refined oils have been explored in laboratory-scale and industrial refining processes $[16,17]$. Analyses of polar compounds in crude oils and in samples taken at different stages of the refining process indicated that the quality of an initial crude oil could be deduced from the resulting refined oil by virtue of certain markers of oxidative and hydrolytic alterations. On one hand, compounds of higher MW than that of oxidized triglyceride monomers are formed, mainly as a consequence of the high temperature in the deodorization step. On the other hand, oxidized triglycerides and diglycerides remain after refining, so their level is a measure of oxidation and hydrolysis, respectively, regardless of whether the oil is crude or refined. Finally, the peak including fatty acids and the polar unsaponifiable fraction decreases due to the neutralization step.

\section{c) Characterization of virgin oils}

Although good results have been obtained through direct analysis of total oils by HPSEC for characterization of virgin oils [18], improved evaluation of minor compounds can be achieved by previous elimination of nonpolar triglycerides, especially for application to oils containing very low levels of polar glyceridic compounds $[19,20]$. By using the combined methodologies detailed above, we have found that the absence of dimers is the most useful parameter for the characterization of virgin oils, while the occurrence of dimers plus a high ratio of diglycerides/fatty acids are those for refined oils. The procedure was later followed to quantitate oxidized triglycerides and diglycerides in different types of olive oils of certified origin [21].

\section{d) Follow-up of early oxidation stages}

Along with the well-established methods which provide information on primary and secondary oxidation products, HPSEC offers a complementary technique for the evaluation of oxidized compounds originating at low temperatures. In

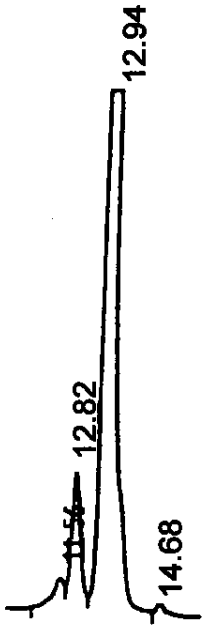

(A)

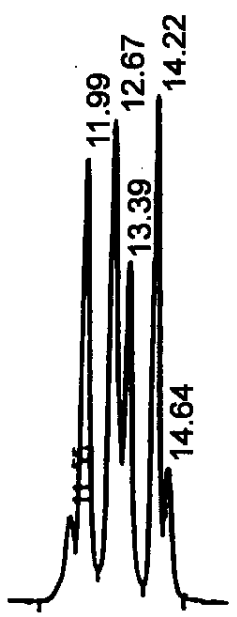

(B)
Figure 3. HPSEC chromatograms of an used frying oil: Total oil (A) and polar compounds (B). Retention times (min): 11.5, triglyceride oligomers; 12.0, triglyceride dimers; 12.9, total triglyceride monomers in (A) and oxidized triglyceride monomers in (B); 13.4, diglycerides; 14.2, monostearin (internal standard) and 14.6, fatty acids.

view of the particular advantages provided by the combination solid phase extraction-HPSEC, this methodology is currently selected to study the useful storage life and efficacy of antioxidants in model systems, oils and fatty foods $[7,22]$.

Figure 4 shows a representative profile of the progress of oxidation for trilinolein samples at $60{ }^{\circ} \mathrm{C}$ wherein oxidized monomers, dimers and oligomers were quantitated in polar fractions. In parallel, peroxide values were determined since it is the most widely used method to monitor early oxidation. As can be observed, oxidized monomers showed a gradual increase during the earlier stages of oxidation, in agreement with the rise in peroxide values. Both

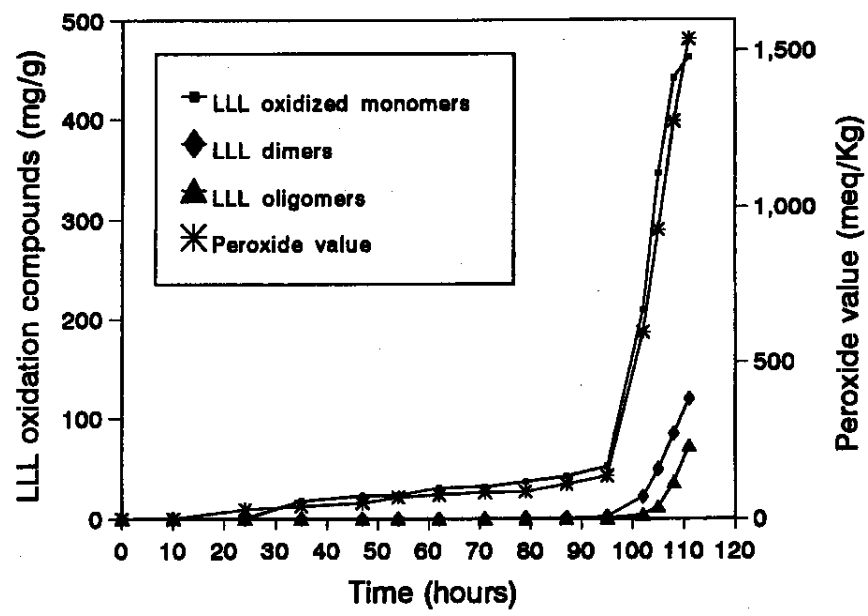

Figure 4. Evolution of oxidized triglyceride monomers, dimers and oligomers; and peroxide value, in samples of trilinolein (LLL) throughout oxidation at $60{ }^{\circ} \mathrm{C}$. 
determinations are consistent since hydroperoxides are the primary oxidation products formed and during the early stages of oxidation, the peak of oxidized triglyceride monomers consist primarily of hydroperoxides. However, while peroxides are intermediate products, the resulting oxygenated side products of the same chain length as the parent hydroperoxide are also included in the peak of oxidized monomers. Therefore, quantitation of this group of compounds is of great utility not only to detect initially formed oxidized products - even before rancidity - but also to follow oxidation during further stages. At a certain point, oxidation is accelerated as shown by a rapid increase of oxidized monomers and initiation of polymerization, thus indicating the end of the induction period. Overall, this analytical approach allows concomitant evaluation of primary and secondary oxidation compounds thus enabling the degree of oxidation to be determined at any point during the course of oxidation.

\section{Methyl ester derivatives of fats and oils}

Complementary information to that obtained by the methodologies outlined above can be attained by analyzing specifically the oxidized and polymeric fatty acyl groups included in triglyceride molecules through preliminary transesterification of samples and application of a similar analytical scheme to the fatty acid methyl esters [23]. In this approach, the range of molecular weights of the compounds separated decreases, and it is not easy to find a suitable internal standard. Hence quantitative data have been obtained here only on the basis of gravimetric determinations.

Briefly, $1 \mathrm{~g}$ of methyl esters are separated by silica column chromatography, using $150 \mathrm{~mL}$ hexane:diethyl ether 88:12 and $150 \mathrm{~mL}$ diethyl ether to elute the nonpolar and polar fractions, respectively. Both fractions are gravimetrically determined and further analyzed by HPSEC. Thus, the combined chromatographic analysis permits quantitation of five types of fatty acyl groups: unoxidized monomers and nonpolar dimers (representative of thermal degradation since no oxygen is involved in their structure) in the first fraction, while oxidative dimers are determined separately in the second fraction, along with oxidized monomers and oligomers.

\section{a) Analysis of used frying fats and oils}

Complementary analysis of heated fats after transesterification when combined with evaluation of polar compounds in the original sample is of great use for acquiring a broader information of fat alteration. Special focus has been placed on evaluation of used frying fats collected by Food Inspection Services and, particularly on those samples with polar compound levels close to the limit for frying fat rejection [24].

While detection of hydrolytic alteration can be only attained through HPSEC analysis of polar fractions of original oil samples, differences in thermoxidative degradation are better reflected in the values of altered fatty acid methyl esters since exclusively the amount of fatty acyl groups affected are measured. Also, some insight into the complexity of the triglyceride polymer structure can be revealed by comparing triglyceride and methyl ester dimers and oligomers values. To illustrate this, table I shows selected results corresponding to three samples of used frying sun-
Table I. Quantitation of triglyceride (TG) oligomers and dimers, and fatty acid methyl ester (FAME) oligomers and dimers (wt\% on total sample) in used frying higholeic sunflower oils.

\begin{tabular}{llcc}
\hline \multicolumn{2}{l}{ Sample } & Oligomers & Dimers \\
\hline A & TG $^{\mathrm{a}}$ & 2.1 & 5.7 \\
& FAME $^{\mathrm{b}}$ & 0.4 & 3.2 \\
$\mathrm{~B}$ & $\mathrm{TG}$ & 5.8 & 8.6 \\
& $\mathrm{FAME}$ & 1.0 & 5.0 \\
$\mathrm{C}$ & $\mathrm{TG}$ & 13.3 & 12.1 \\
& FAME & 2.5 & 7.8 \\
\hline
\end{tabular}

a Determined in oil sample.

b Determined after conversion of oil to fatty acid methyl esters.

flower oil. The low fatty acid oligomers- to- triglyceride oligomers ratios in contrast to the fatty acid dimers-to-triglyceride dimers ratios give evidence of the considerable contribution of dimeric linkages to the structures of trimeric and higher oligomeric triglycerides.

\section{b) Nutritional evaluation of used frying fats and oils}

Given that the products of fat digestion ultimately absorbed are majoritarily the fatty acids released by the action of pancreatic lipase, the analytical approach starting from fatty acid methyl esters has offered an excellent tool to examine the digestibility coefficients of each group of alteration compounds in animal studies, showing especially high values (around $80 \%$ ) for the group of oxidized fatty acid monomers [25]. Poor digestibility of polymers could be due to low activity of pancreatic lipase on triglyceride polymers or to poor absorption of the fatty acid polymers released. In this line, direct application of HPSEC to thermoxidized oils subjected to in vitro hydrolysis showed the difficulties involved in the enzymatic action on complex glycerides [26,27] while analyses of fecal lipids confirmed that a considerable fraction of high MW compounds ingested remained non-hydrolyzed [28].

\section{References}

1. Dobarganes, M. C.; Márquez-Ruiz, G. Size exclusion chromatography in the analysis of lipids. In: Advances in Lipid Methodology-Two, Christie, W. W. Ed. The Oily Press, Dundee, 1993; pp 113-137.

2. Dobarganes, M. C.; Márquez-Ruiz, G. High-performance sizeexclusion chromatography applied to the analysis of edible fats. In: New Trends in Lipid and Lipoprotein Analyses, Sebedio, J. L.; Perkins, E. G. Eds. AOCS Press, Champaign, 1995; pp 81-92.

3. Márquez-Ruiz, G.; Dobarganes, M. C. Analysis of lipid oxidation products by combination of chromatographic techniques. In: New Techniques and Applications in Lipid Analysis, McDonald, R. E.; Mossoba, M. Eds., AOCS Press, Champaign, 1997; pp 216-233.

4. Perrin, J. L.; Redero, F.; Prevot, A. Dosage rapide des polymères de triglycérides par chromatographie d'exclusion, Rev. Franc. Corps Gras 1984, 31, 131-133.

5. Burkow, I. C.; Henderson, R. J; Isolation and quantification of polymers from autoxidized fish oils by high-performance size- 
exclusion chromatography with an evaporative mass detector, J. Chromatogr. 1991, 552, 501-506.

6. Wolff, J. P.; Mordret, F. X.; Dieffenbacher, A. Determination of polymerized triglycerides in oils and fats by high performance liquid chromatography, Pure Appl. Chem. 1991, 63, 1163-1171.

7. Márquez-Ruiz, G.; Martín-Polvillo, M.; Dobarganes, M. C. Quantitation of oxidized triglyceride monomers and dimers as an useful measurement for early and advanced stages of oxidation, Grasas y Aceites 1996, 47, 48-53.

8. Haumann, B. F. Fat replacers: Identifying valid analytical methods, INFORM 1993, 4, 1226-1235.

9. Ríos, J. J.; Pérez-Camino, M. C.; Márquez-Ruiz, G.; Dobarganes, M. C. Isolation and characterization of sucrose polyesters, J. Am. Oil Chem. Soc. 1994, 71, 385-390.

10. Márquez-Ruiz, G.; Pérez-Camino, M. C.; Ríos, J. J.; Dobarganes, M. C. Characterization of sucrose polyesters-triacylglycerols mixtures, J. Am. Oil Chem. Soc. 1994, 71, 10171020.

11. Waltking, A. E.; Wessels, H. Chromatographic separation of polar and non-polar components of frying fats, J. Assoc. Off. Anal. Chem. 1981, 64, 1329-1330.

12. Firestone, D. Regulation of frying fats and oils. In: Deep Frying: Chemistry, Nutrition and Practical Applications, Perkins, E. G.; Erickson, M. D., Eds., AOCS Press, Champaign, 1996; pp 323-334.

13. Dobarganes, M. C.; Pérez-Camino, M. C.; Márquez-Ruiz, G. High performance size exclusion chromatography of polar compounds in heated and non-heated fats, Fat. Sci. Technol. 1988, 90, 308-311.

14. Márquez-Ruiz, G.; Jorge, N.; Martín-Polvillo, M.; Dobarganes, M. C. Rapid, quantitative determination of polar compounds in fats and oils by solid-phase extraction and exclusion chromatography using monostearin as internal standard, $J$. Chromatogr. 1996, 749, 55-60.

15. Arroyo, R.; Cuesta, C.; Garrido-Apolonio, C.; López-Varela; Sánchez-Muñiz, F. J. High-performance size-exclusion chroamtography studies on polar components formed in sunflower oils used for frying, J. Am. Oil Chem. Soc. 1992, 69, 557-563.

16. Dobarganes, M. C.; Pérez-Camino, M. C.; Márquez-Ruiz, G.; Ruiz-Méndez, M. V. New analytical possibilities in quality evaluation of refined oils. In: Edible Fats and Oils Processing: Basic Principles and Modern Practices, Erikson, D. R. Ed., AOCS Press, Champaign, 1989; pp 427-429.
17. Hopia, A. Analysis of high molecular weight autoxidation products using high performance size exclusion chromatography: II. Changes during processing, Food Sci. Technol. 1993, 26, 568-571.

18. Gomes, T. Oligopolimeri in oli vegetali sottoposti a trattamenti termici, Riv. Ital. Sostanze Grasse 1988, 65, 433-438.

19. Dobarganes, M. C.; Pérez-Camino, M. C.; Márquez-Ruiz, G. Application of minor glyceridic component determination to the evaluation of olive oil. In: Actes du Congrès International "Chevreul » pour l'étude des corps gras, Vol. 2 , ETIG, Paris, 1989; pp 578-584.

20. Pérez-Camino, M. C.; Ruiz-Méndez, M. V.; Márquez-Ruiz, G.; Dobarganes, M. C. Aceites de oliva vírgenes y refinados: diferencias en componentes menores glicerídicos, Grasas y Aceites 1993, 44, 91-96.

21. Gomes, T. Oligopolymer, diglyceride and oxidized triglyceride contents as measures of olive oil quality, J. Am. Oil Chem. Soc. 1992, 69, 1219-1223.

22. Martín-Polvillo, M.; Márquez-Ruiz, G.; Jorge, N.; RuizMéndez, M. V.; Dobarganes, M. C. Evolution of oxidation during storage of crisps and french fries prepared with sunflower oil and high oleic sunflower oil, Grasas y Aceites 1996, 47, 54-58.

23. Márquez-Ruiz, G.; Pérez-Camino, M. C.; Dobarganes, M. C. Combination of absorption and size-exclusion chromatography for the determination of fatty acid monomers, dimers and polymers, J. Chromatogr. 1990, 514, 37-44.

24. Márquez-Ruiz, G.; Tasioula-Margari, M.; Dobarganes, M. C. Quantitation and distribution of altered fatty acids in frying fats, J. Am. Oil Chem. Soc. 1995, 72, 1171-1176.

25. Márquez-Ruiz, G.; Pérez-Camino, M. C.; Dobarganes, M. C. Digestibility of fatty acid monomers, dimers and polymers in the rat, J. Am. Oil Chem. Soc. 1992, 69, 930-934.

26. Márquez-Ruiz, G.; Pérez-Camino, M. C.; Dobarganes, M. C. In vitro action of pancreatic lipase on complex glycerides from thermally oxidized oils, Fat Sci. Technol. 1992, 94, 307-312.

27. Henderson, R. J.; Burkow, I. C.; Millar, R. M. Hydrolysis of fish oils containing polymers of triacylglycerols by pancreatic lipase in vitro, Lipids 1993, 28, 313-319.

28. Márquez-Ruiz, G.; Pérez-Camino, M. C.; Dobarganes, M. C. Evaluation of hydrolysis and absorption of thermally oxidized olive oil in non-absorbed lipids in the rat, Ann. Nutr. Metab. 1993, 37, 121-128. 